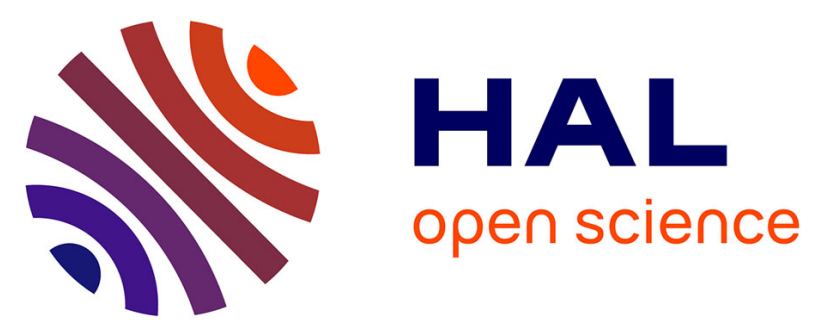

\title{
Weakly supervised learning from scale invariant feature transform keypoints: an approach combining fast eigendecompostion, regularization, and diffusion on graphs
}

Youssef Chahir, Bouziane Abderraouf, Mostefai Messaoud, Adnan Al Alwani

\section{To cite this version:}

Youssef Chahir, Bouziane Abderraouf, Mostefai Messaoud, Adnan Al Alwani. Weakly supervised learning from scale invariant feature transform keypoints: an approach combining fast eigendecompostion, regularization, and diffusion on graphs. Journal of Electronic Imaging, 2014, 23 (1), pp.13. 10.1117/1.JEI.23.1.013009 . hal-01168415

\section{HAL Id: hal-01168415 \\ https://hal.science/hal-01168415}

Submitted on 18 Sep 2018

HAL is a multi-disciplinary open access archive for the deposit and dissemination of scientific research documents, whether they are published or not. The documents may come from teaching and research institutions in France or abroad, or from public or private research centers.
L'archive ouverte pluridisciplinaire HAL, est destinée au dépôt et à la diffusion de documents scientifiques de niveau recherche, publiés ou non, émanant des établissements d'enseignement et de recherche français ou étrangers, des laboratoires publics ou privés. 


\title{
Weakly supervised learning from scale invariant feature transform keypoints: an approach combining fast eigendecompostion, regularization, and diffusion on graphs
}

\author{
Youssef Chahir, ${ }^{a}$ Abderraouf Bouziane,,${ }^{a, b, *}$ Messaoud Mostefai, ${ }^{b}$ and Adnan Al Alwani ${ }^{a}$ \\ ${ }^{a}$ GREYC-UMR CNRS 6072 Campus II-BP 5186, Université de Caen 14032 Caen Cedex, France \\ bUniversity of Bordj Bou Arreridj, MSE Laboratory, 34000, Algeria
}

\begin{abstract}
We propose a unified approach to propagate knowledge into a high-dimensional space from a small informative set, in this case, scale invariant feature transform (SIFT) features. Our contribution lies in three aspects. First, we propose a spectral graph embedding of the SIFT points for dimensionality reduction, which provides efficient keypoints transcription into a Euclidean manifold. We use iterative deflation to speed up the eigendecomposition of the underlying Laplacian matrix of the embedded graph. Then, we describe a variational framework for manifold denoising based on $p$-Laplacian to enhance keypoints classification, thereby lessening the negative impact of outliers onto our variational shape framework and achieving higher classification accuracy through agglomerative categorization. Finally, we describe our algorithm for multilabel diffusion on graph. Theoretical analysis of the algorithm is developed along with the corresponding connections with other methods. Tests have been conducted on a collection of images from the Berkeley database. Performance evaluation results show that our framework allows us to efficiently propagate the prior knowledge.
\end{abstract}

Keywords: high-dimensional data; graph-based learning; spectral graph embedding; regularization on graphs; classification; scale invariant feature transform features.

\section{Introduction}

Graph-based learning algorithms have spread in recent years, where initial information is asked to the user to highlight critical points of the considered space, subject to exploration. Consequently, the result is initialization dependent. How to avoid manual interactions with the user and efficiently propagate initial knowledge will be of a great interest in computer vision field and its related areas, where identification of objects in images is a challenging research subject. In this case, a family of segmentation algorithms have been developed, where the image is modeled by a graph and the object to be extracted is one for which a certain form of energy functional is minimum. ${ }^{1,2}$ Different forms of energy functional have been developed. In Ref. 3, the authors first use patches of different shapes and different sizes to extract different noise-robust features and then information theorybased measures are computed and minimized. In Ref. 4, the energy functional to be optimized is defined using the geodesic-distance combined with contours information, where the geodesic-distance region information is reevaluated according to the color model deduced by seeds introduced by the user.

On another plan, two main approaches can be distinguished, whereas the segmentation process is either done automatically or guided by information provided by the user regarding the object of interest. To perform automatic segmentation, the authors in Ref. 5 use the camera fixation

*Address all correspondence to: Abderraouf Bouziane, E-mail: bouziane .abderraouf@gmail.com point on the object of interest to deduce a color model and a surrounding contour. Iterating this process minimizes the graph-cut energy and enhances the segmentation result. In a similar approach, the energy functional to minimize in Ref. 6 is expressed by an active contour model and the image is divided into two regions using a graph-cut. The object of interest is obtained by repeating this process until convergence to a defined threshold.

In the interactive approach, the user supplies initial seeds to differentiate between the object of interest and the background, hence, avoiding object/background estimations, and depending on where the seeds are placed, two types of initialization are studied: one relying on the definition of parts of the object and parts of the background, ${ }^{1,7}$ and the other one starts with an initial contour, ${ }^{8}$ which may either enclose the object, part of the object, ${ }^{9}$ or delimit the border between the object and the background. ${ }^{10}$ However, this approach is not effective if the segmentation process will be integrated into an automated framework. Moreover, the result is initialization dependent. Indeed, it is possible that, due to the complexity of the image, among the seeds introduced by the user to designate parts of the object (respectively, background), some of them are of the same class of the background (resp. object), or simply, the seeds are not representative enough.

To avoid manual interactions, we investigate in this paper the use of the image keypoints as initial seeds to denote the ground truth. These are automatically extracted and they are first categorized and then diffused on the image to extract the 
object of interest. A variety of keypoint descriptors have been proposed, such as Harris corner detector, ${ }^{11}$ the scale invariant feature transform (SIFT), ${ }^{12}$ gradient location and orientation histogram, ${ }^{13}$ and difference of means. ${ }^{14}$ The SIFT descriptors are currently most widely used in computer vision applications due to the fact that they are highly distinctive and invariant to scale, rotation, and illumination changes. In addition, they are relatively easy to extract and to match against a large set of local features. Several improvements of SIFT features are proposed, including affine scale invariant feature transform (ASIFT) ${ }^{15}$ and PCA-SIFT, ${ }^{16}$ which applies principal components analysis to the SIFT descriptor in order to reduce the SIFT feature descriptor dimensionality from 128 to 36 .

However, each keypoint is described by a considerable number of attributes and then distributed in a high-dimensional space. To overcome this constraint, in our approach we propose to construct a similarity graph over the SIFT descriptors. The eigendecomposition of the Laplacian matrix, associated to this graph, allows us to identify the dimensions that are carrying the relevant information. A Euclidean distance is then defined on these dimensions as proposed in Ref. 17, and the keypoints can be, therefore, classified through agglomerative categorization. However, if the image reveals a high number of keypoints, which is often the case, the size of the Laplacian matrix may slow down the calculation of eigenvalues and their correspondent eigenvectors and the segmentation algorithm, therefore, becomes very costly in computation.

Many works in the literature, including the matrix perturbation theory ${ }^{18,19}$ and the Nyström method, ${ }^{20,21}$ have been proposed to accelerate the spectral decomposition via the approximation of the eigenvalues and their correspondent eigenvectors. In Ref. 22, the approximation to the leading eigenvector is based on a linear perturbation analysis of matrices that are nonsparse, nonnegative, and symmetric. Huang et al. ${ }^{23}$ studied the effects of data perturbation on the performance of spectral clustering and its relation with the perturbation of the eigenvectors of the Laplacian matrix. In its turn, the Nyström method has been very successful. In Ref. 24, the authors show its use to approximate the eigendecomposition of the Gram matrix in order to speed up kernel machines. In Ref. 25, Fowlkes et al. present a technique for the approximate solution of spectral partitioning for image and video segmentation based on the Nyström extension. Other variants of the Nyström method are presented in Refs. 26 and 27

Practically, one can use the subjective scree-test of Cattell ${ }^{28}$ to determine the most important $k$ 'th dimensions that enclose the pertinent information. This criterion is based on the analysis of differences between consecutive eigenvalues, where a breakpoint would be located where there is the biggest change in the slope of the curve of eigenvalues. The first $k$ 'th eigenvalues then correspond to the number of dimensions to retain (see Ref. 29 for detail). Another simple way is to consider the first dominant eigenvalues for which their sum is greater than a defined threshold (e.g., $\geq 80 \%$ ).

\subsection{Outline}

The basic idea of our paper is to show how it is possible to learn from a restricted data set and propagate the acquired knowledge to a high-dimensional database. To evaluate the effectiveness of our approach, in this article we focus on the case of image segmentation. The proposed framework operates in two phases. In the first phase, seeds are automatically identified and classified, and in the second phase, a propagation of these seeds on the graph allows to highlight the object of interest. The following steps outline our approach:

- A set of SIFT keypoints is extracted from the image and used to construct a visual similarity graph. A spectral embedding of this graph is performed to define a Euclidean reduced space. ${ }^{17}$ To speed up the spectral graph embedding, we propose to use the power iteration algorithm combined with the deflation method to compute the first $k$ 'th largest eigenvalues and their corresponding eigenvectors, which are often well suited to define the new pose space basis. To help the categorization process, we perform a discrete regularization of the graph constructed over the SIFT keypoints expressed in their new coordinates. Thus, the clustering is done in the inferred regularized Euclidean manifold.

- At this step, a new graph is constructed over the image. It will contain labeled vertices and unlabeled ones. By using our multilabel propagation algorithm, an energy functional is formulated and minimized. The objects of interest are then extracted when some conditions are satisfied.

It is worth mentioning that at each step, we will use a different graph, i.e., a graph over the SIFT descriptors (feature space) to allow spectral embedding and a graph in the measure space (the embedded space) to manifold regularization in order to enhance the accuracy of the keypoints categorization. Once the keypoints are labeled (classified), a final graph is constructed over the whole image (initial input data). It will contain labeled vertices and unlabeled ones. It will be used to propagate the information of the labeled keypoints in their neighborhoods. A graphical illustration of our framework is presented in Fig. 1.

The rest of the paper is organized as follows. Section 2 gives an overview of our spectral embedding framework. In Sec. 3, we explain how to speed up eigendecomposition. In Sec. 4, we present a discrete regularization on the graph in the embedded space to enhance data robustness. In Sec. 5, a multilabel diffusion algorithm is detailed along with connections with other methods. Experimental results are presented and commented in Sec. 6. In Sec. 7, we conclude our paper and discuss future extension.

\section{Spectral Embedding Framework}

The goal of the spectral analysis of the image to be segmented is to find an optimized pose space where relevant information is captured and similarity between pixels can be easily expressed. With this intention, a set of SIFT keys $\mathcal{X}=\left\{P t_{1}, P t_{2}, \ldots, P t_{n}\right\}$ is extracted from an image through the local invariant feature extraction ${ }^{12}$ (see Fig. 2).

Each SIFT key $P t_{i}=\left(X_{i}, R_{i}, U_{i}\right)$ is described by its twodimensional location in the image $X_{i}=\left(x_{i}, y_{i}\right)$, its gradient magnitude and orientation $R_{i}=\left(r_{i}, a_{i}\right)$, and a descriptor vector $U_{i}=\left(u_{i, 1}, \ldots, u_{i, 128}\right)$, which represents the local texture in the image. From the set $\mathcal{X}=\left\{P t_{1}, P t_{2}, \ldots, P t_{n}\right\} \in$ $\mathbb{R}^{l}$, we develop an appropriate Euclidean mapping 


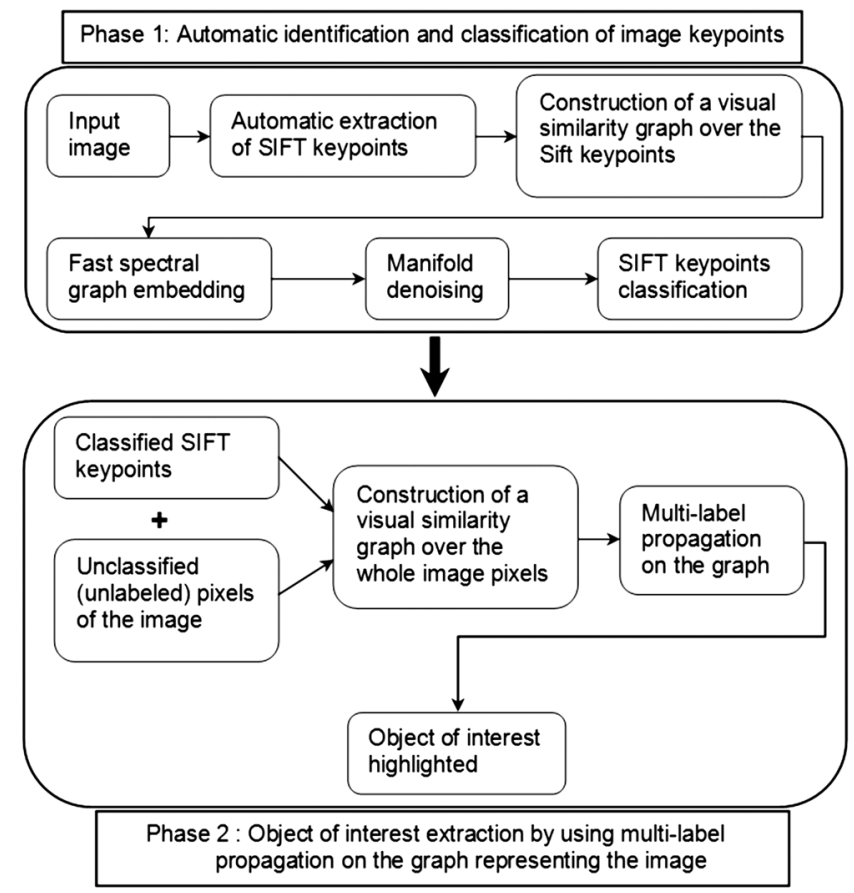

Fig. 1 Our diffusion framework.

$\mathcal{Y}=\left\{y_{1}, y_{2}, \ldots, y_{n}\right\} \in \mathbb{R}^{m}$ representing the pose space $(m \ll l)$. To do this, we build an undirected graph $G$ on $\mathcal{X}$ and we learn a kernel matrix that respects the provided side-information as well as the local/nonlocal geometry of the SIFT features. Through the eigendecomposition of the matrix associated to the random walks on $G$, we define a diffusion distance $D\left(y_{i}, y_{j}\right)$.

\subsection{Random Walks on Graph}

Let $G=(V, E, w)$ be a weighted undirected graph, which is a finite set of vertices $V=\left\{v_{1}, v_{2}, \ldots, v_{n}\right\}$ connected by a finite set of edges $E \subseteq V \times V$. Let $f(v)$ be a function defined on the vertex $v$ in a $K$-dimension space and represented by the tuple $\left\{f_{1}, f_{2}, \ldots, f_{K}\right\} \in \mathbb{R}^{K}$. We denote by $u \sim v$ the fact that the node $u$ belongs to the $\varepsilon$-neighborhood of $v$ $\left[u \in \mathcal{N}_{\varepsilon}(v)\right]$, which is defined by

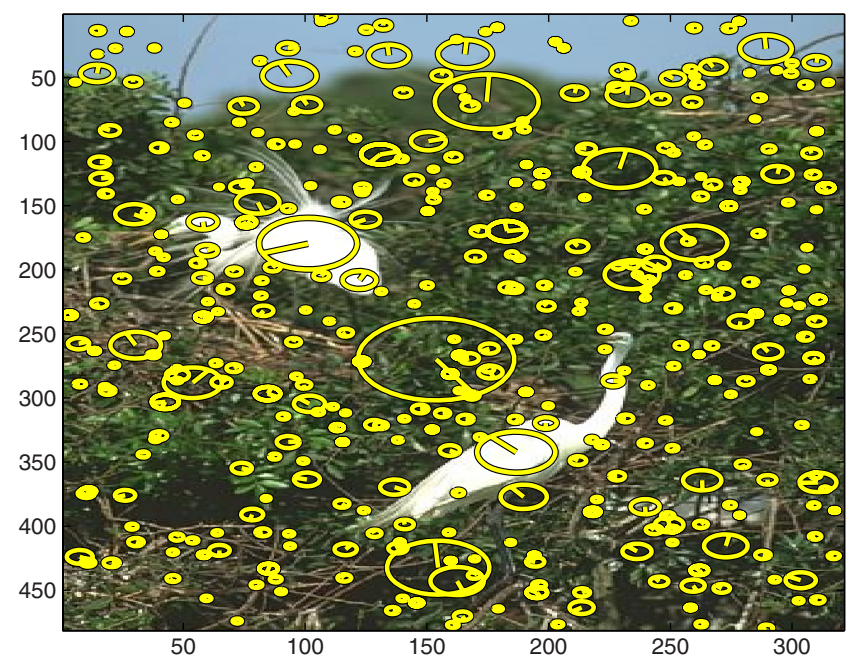

Fig. 2 Scale invariant feature transform keypoint extraction.

$$
\begin{aligned}
& \mathcal{N}_{\varepsilon}(v) \\
& \quad=\left\{u \in V, f(u)=\left(f_{1}^{\prime}, \cdots, f_{K}^{\prime}\right) /\left|f_{i}-f_{i}^{\prime}\right| \leq \varepsilon_{i}, 0<i \leq K\right\}
\end{aligned} .
$$

$\mathcal{N}_{\varepsilon}(v)$ includes all vertices close and similar to the vertex $v$. Also, we define a function $F$ on the patch surrounding the vertex $v$ as follows:

$$
F(v)=\left\{f(u), u \in \mathcal{N}_{\varepsilon}(v)\right\} .
$$

For example, for a sequence of images, $f(v)$ may be the spatiotemporal attributes of a vertex $v . F(v)$ can represent the characteristics of the vertex in its neighborhood: a pattern projecting a composite element or a visual vocabulary.

We use a Gaussian kernel $W$ to define the weight function and to give a measure of the similarity between a vertex and its neighbors. This weight function can incorporate local and nonlocal features and is defined by

$$
\begin{aligned}
& w(u, v)
\end{aligned}
$$

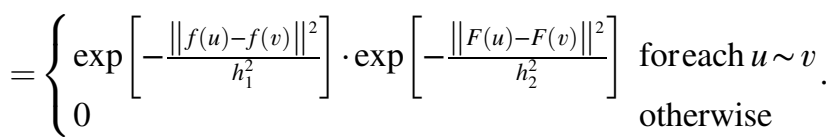

$h_{i}$ can be estimated using the standard deviation depending on the variations of $|f(u)-f(v)|$ and $\|F(u)-F(v)\|$ over the graph, respectively. So, given the scale parameter $h_{i}>0, w_{h_{i}}(u, v) \rightarrow 0$ when $\|.\| \gg h_{i}$ and $w_{h_{i}}(u, v) \rightarrow 1$ when $\|.\| \ll h_{i}$.

We recall that the degree $d(v)$ of a node $v$ and the volume $\operatorname{Vol}(G)$ of $G$ are defined, respectively, by

$d(v)=\sum_{u \sim v} w(u, v), \quad$ and $\quad \operatorname{Vol}(G)=\sum_{v \in V} d(v)$.

The graph $G$ reflects the knowledge of the local/nonlocal geometry of the data set $\mathcal{X}$ and is seen as a Markov chain; a random walk on this graph is the process that begins at some vertex $u$ and at each time step, moves to another one $v$ with a probability proportional to the weight of the corresponding edge. Thus, one can define the diffusion on $G$ as the set of the possible visited vertices starting from a given one, where a transition is made in one time step from a vertex $u$ toward another vertex $v$ chosen randomly and uniformly among its neighborhood with the probability

$p^{(1)}(u, v)=\operatorname{Pr}\left(X_{t+1}=v \mid X_{t}=u\right)=\frac{w(u, v)}{d(u)}$.

The transition matrix $P$ on $G$ given by $P=$ $\left\{p^{(1)}(u, v) \mid u, v \in V, u \sim v\right\}$ explicits all possible one time step transitions and, therefore, provides the first-order information of the graph structure.

Let $P^{t}$ be the $t$ power of the matrix $P$, which denotes the set of all transition probabilities $p^{(t)}(u, v)$ of going from one vertex $u$ to another one $v$ in $t$ time steps. So, on the graph $G$, $p^{(t)}(u, v)$ reflects all paths of length $t$ between the vertex $u$ and the vertex $v$. This $t$-time steps transition probability satisfies the Chapman-Kolmogorov equation that for any $k$ such that $0<k<t$, 


$$
\begin{aligned}
p^{(t)}(u, v) & =\operatorname{Pr}\left(X_{t}=v \mid X_{0}=u\right) \\
& =\sum_{y \in V} p^{(k)}(u, y) \cdot p^{(t-k)}(y, v) .
\end{aligned}
$$

It has been shown in Ref. 30 that

$$
\lim _{t \rightarrow \infty} p^{(t)}(u, v)=\frac{d(v)}{\operatorname{Vol}(G)} .
$$

\subsection{Diffusion Maps-Based Clustering}

For clustering purposes, a connection with the spectral decomposition of $P^{t}$ is made (see Ref. 31 for detail) to generate Euclidean coordinates for the low-dimensional representation of the vertices of the graph $G$ at the time $t$, where, for each vertex, these coordinates are given by

$\Psi_{t}(u)=\left[\lambda_{1}^{t} \psi^{1}(u), \lambda_{2}^{t} \psi^{2}(u), \ldots, \lambda_{n}^{t} \psi^{n}(u)\right]^{T}$.

$\left\{\lambda_{i}^{t}, \psi^{i}(u)\right\}$ are the eigenvalues and the eigenvectors associated with the normalized graph Laplacian of $P^{t}$. They correspond to the nonlinear embedding of the vertices of the graph $G$ onto the new Euclidean pose space. Thus, the diffusion distance $D_{t}^{2}(u, v)$ between the nodes of the graph $G$ can be expressed in the embedded space by

$$
D_{t}^{2}(u, v)=\sum_{i \geq 1} \lambda_{i}^{t 2}\left[\psi_{i}(u)-\psi_{i}(v)\right]^{2}=\left\|\Psi_{t}(u)-\Psi_{t}(v)\right\|^{2} .
$$

We note, in particular, that this new distance depends on the time parameter $t$, which is considered here as a precision parameter, where, for large values, more information on the structure of the graph are captured. Based on their new coordinates, a classification of the SIFT keypoints can now be easily performed by using an agglomerative categorization algorithm based on the Euclidean distance defined in Eq. (9).

\section{Eigendecomposition Speed Up}

The eigenvalues of the matrix $P^{t}$ are obtained by solving its characteristic equation

$\lambda^{n}+c_{n-1} \lambda^{n-1}+c_{n-2} \lambda^{n-2}+\ldots+c_{0}=0$.

But for large values of $n$, this equation is difficult and time-consuming to solve. An alternative method for approximating these eigenvalues is to use the power iteration algorithm and the deflation method to find the dominant eigenvector and the corresponding eigenvalue, exploiting the fact that eigendecomposition of the matrix $P^{t}$ provides a set of eigenvalues ordered as follows:

$1=\left|\lambda_{0}\right| \geq\left|\lambda_{1}\right| \geq\left|\lambda_{2}\right| \geq \ldots\left|\lambda_{n}\right| \geq 0$.

Indeed, the power iteration algorithm is a simple method for computing the largest eigenvector because it accesses to a matrix only through its multiplication by vectors. This property is particularly interesting in the case of large matrices. And the deflation method allows us to remove at each iteration the largest eigenvalue and rearrange the matrix so that the largest eigenvalue of the new matrix will be the second largest eigenvalue of the original matrix. This process can be repeated to compute the remaining eigenvalues.
Algorithm 1 The power iteration algorithm.

Require: $V^{0}$, a nonzero vector in $\mathbb{R}^{n}$

Ensure: An approximation to the dominant eigenvector

1: while $\left\|V^{k}-V^{k-1}\right\| /\left\|V^{k}\right\| \geq \varepsilon$ do

2: Set $X^{k}=P^{t} V^{k-1}$

3: Set $\alpha^{k}=$ the largest element of $X^{k}$ (in absolute value)

4: Set $V^{k}=X^{k} / \alpha^{k}$

5: end while

6: return $V^{k}$, an approximation to the leading eigenvector of $P^{t}$

\subsection{Power Iteration Algorithm}

To have a good approximation of the dominant eigenvector of the matrix $P^{t}$, one can choose an initial approximation $V^{0}$, which must be a nonzero vector in $\mathbb{R}^{n}$ so that the sequence of its multiplication by $P^{t}$ will converge to the leading eigenvector. Algorithm 1 summarizes the power iteration method. In algorithm dividing by $\alpha$ in step 4 is to scale down each approximation before proceeding to the next iteration in order to avoid reaching vectors whose components are too large (or too small). For large powers, $k$, we will obtain a good approximation to the dominant eigenvector. Indeed, since $P^{t}$ is a symmetric positive-semidefinite matrix, it has a basis of orthonormal eigenvectors $\left\{\psi_{i}\right\}$, and the initial approximation $V^{0}$ can then be written as

$V^{0}=\sum_{i=1}^{n} \beta_{i} \psi_{i}, \quad \beta_{i} \in \mathbb{R}$.

Suppose that $\psi_{1}$ is the eigenvector corresponding to the dominant eigenvalue $\lambda_{1}$; then we can easily write

$$
\begin{aligned}
V^{k} & =P_{t}^{k} V^{0}=\sum_{i=1}^{n} \beta_{i} P_{t}^{k} \psi_{i}=\sum_{i=1}^{n} \beta_{i} \lambda_{i}^{k} \psi_{i} \\
& =\beta_{1} \lambda_{1}^{k}\left\{\psi_{1}+\ldots+\sum_{i=2}^{n} \frac{\beta_{i}}{\beta_{1}}\left[\frac{\lambda_{i}}{\lambda_{1}}\right]^{k} \psi_{i}\right\} .
\end{aligned}
$$

Since $\lambda_{1}$ is the dominant eigenvalue, it follows that

$\lambda_{i} / \lambda_{1}<1$, and $\forall i>1, \lim _{k \rightarrow \infty}\left(\lambda_{i} / \lambda_{1}\right)^{k} \rightarrow 0$. We then deduce that $P_{t}^{k} V^{0} \approx \beta_{1} \lambda_{1}^{k} \psi_{1}, \beta_{1} \neq 0$, which means that the direction of $V^{k}$ stabilizes to that of $\psi_{1}$, and since $\psi_{1}$ is a dominant eigenvector, it follows that any scalar multiple of $\psi_{1}$ is also a dominant eigenvector. Furthermore, since the eigenvalues of $P^{t}$ are ordered like in Eq. (11), the power method will converge quickly if $\left|\lambda_{1}\right| /\left|\lambda_{2}\right|$ is small and slowly if $\left|\lambda_{1}\right| /\left|\lambda_{2}\right|$ is nearly equal to 1 .

\subsection{Deflation Method}

Once an approximation to the dominant eigenvector $\psi_{i}$ is computed, the Rayleigh quotient provides a correspondingly good approximation to the dominant eigenvalue $\lambda_{i}$, which is given by 
$\lambda_{i}=\frac{\left(P_{i}^{t} \psi_{i}\right)^{T} \cdot \psi_{i}}{\psi_{i}^{T} \psi_{i}}$

To compute the remaining eigenvalues, one can modify the matrix $P_{i}^{t}$ into $P_{i+1}^{t}, \ldots$, as follows:

$P_{i+1}^{t}=P_{i}^{t}-\lambda_{i} \frac{\psi_{i} \psi_{i}^{T}}{\psi_{i}^{T} \psi_{i}}$.

$P_{i+1}^{t}$ has the same eigenvectors and eigenvalues as $P_{i}^{t}$ except that $\lambda_{i}$ is shifted to 0 , leaving the other eigenvalues unchanged. Indeed, for any eigenvector $\psi_{j}, j=$ $(i+1, i+2, \cdots, n)$ of $P^{t}, P_{i+1}^{t}$ satisfies

$$
\begin{aligned}
P_{i+1}^{t} \psi_{j} & =P_{i}^{t} \psi_{j}-\lambda_{i} \frac{\left(\psi_{i} \psi_{i}^{T}\right) \cdot \psi_{j}}{\psi_{i}^{T} \psi_{i}} \\
& =P^{t} \psi_{j}-\lambda_{i} \frac{\psi_{i} \cdot\left(\psi_{i}^{T} \psi_{j}\right)}{\psi_{i}^{T} \psi_{i}} .
\end{aligned}
$$

Since the set of the eigenvectors $\left\{\psi_{i}\right\}$ forms an orthonormal basis (i.e., $\psi_{i}^{T} \psi_{j}=0$ ), $P_{i+1}^{t} \psi_{j}=P_{i}^{t} \psi_{j}$. Thus, the eigenvectors of $P_{i+1}^{t}$ are the same as those of $P_{i}^{t}$ and its eigenvalues are $\lambda_{i+1}, \cdots, \lambda_{n}$. The power method applied to $P_{i+1}^{t}$ will then pick out the next largest eigenvalue $\lambda_{i+1}$.

To determine the principal eigenvalues that gather the relevant information, the eigengap heuristic approach computes the gap between consecutive eigenvalues $\lambda_{k}$ and $\lambda_{k+1}$. The first $\lambda_{k}$ carrying the principal information are those for which $\lambda_{k} \gg \lambda_{k+1}$ (i.e., $\left|\lambda_{k}-\lambda_{k+1}\right|$ is relatively large). Practically, the $6 / 7$ first eigenvalues are sufficient to gather the pertinent information in the reduced space. To verify this method, we have conducted tests on different matrices of different sizes. We were limited to the 10th first important eigenvalues and eigenvectors. For example, in Fig. 3, the eigengap is well observed between the first and the second eigenvalue.

As shown in Fig. 4, there is practically no difference between the eigenvalues using this technique and those using the singular value decomposition (SVD) method.

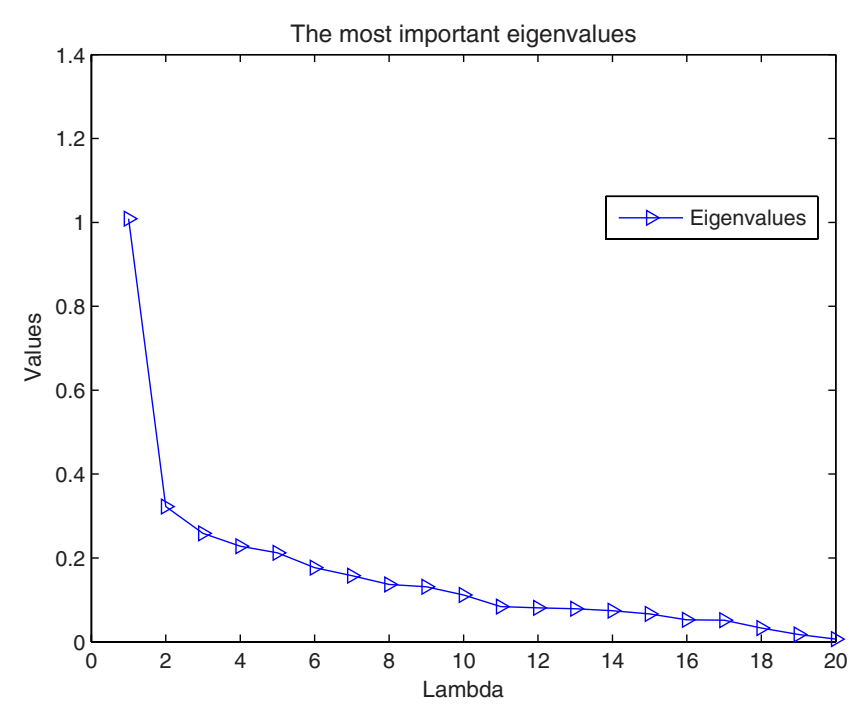

Fig. 3 The most important eigenvalues.

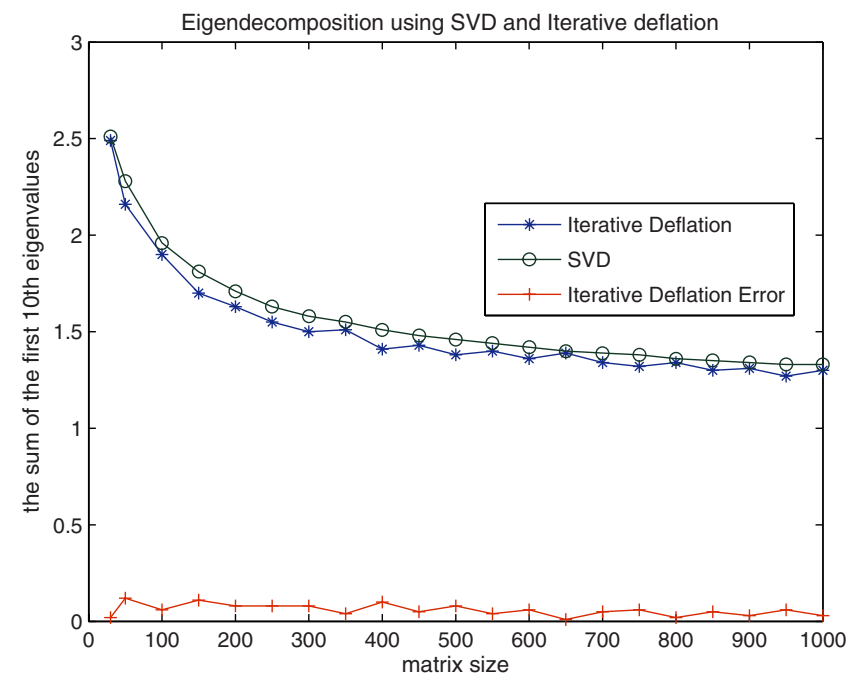

Fig. 4 The sum of the first 10th eigenvalues using SVD and iterative deflation.

However, regarding the computation time (see Fig. 5), it is clear that the iterative deflation approach is more efficient since it can compute only the first ones, while the SVD method has to decompose the whole matrix to extract the considered eigenvalues and eigenvectors and consequently consumes more time.

\section{Manifold Regularization}

Our motivation for this section is to transcribe the variational methods on a discrete graph. To this end, we propose to extend the scope of discrete regularization ${ }^{32}$ to high-dimensional data. We have implemented algorithms for regularization on graphs with $p$-Laplacian, $p \in] 0,+\infty$ [, for denoising and simplification of data in the embedded space. Readers can refer to Ref. 33 for further details on this formalism.

Recall that the function $f^{0}$ is an observation of an original function $f$ affected by noise $n: f^{0}=f+n$. The discrete

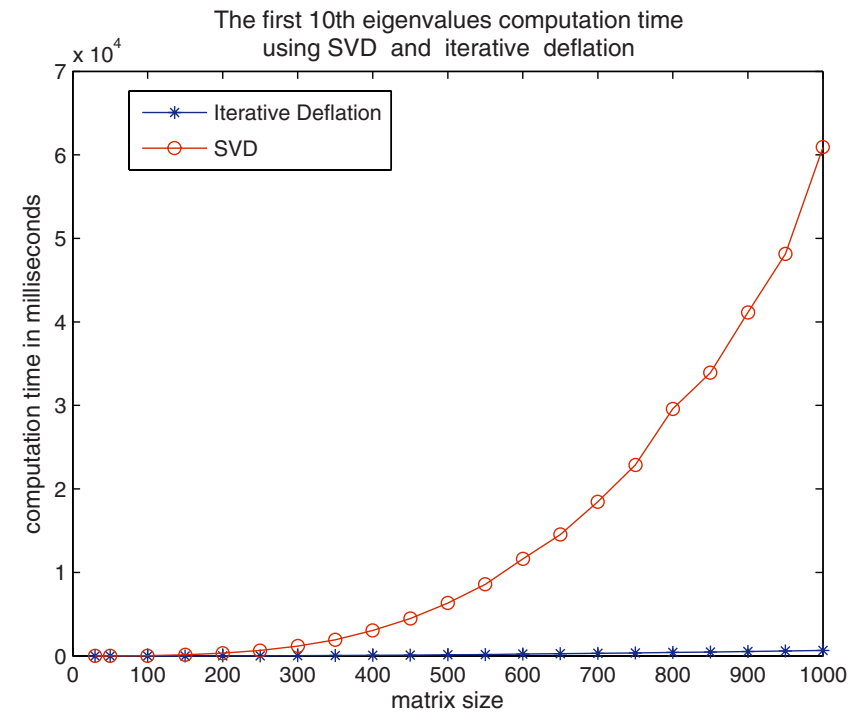

Fig. 5 The first 10th eigenvalues computation time using singular value decomposition and iterative deflation. 
regularization of $f^{0} \in(V)$ using the weighted $p$-Laplacian operator consists of seeking a function $f^{*} \in(V)$, which is not only smooth enough on $G$, but also sufficiently close to $f^{0}$. Variational models of regulation can be formalized by the minimization of two terms of energy using either the isotropic $p$-Laplacian or the anisotropic $p$-Laplacian. The isotropic model gives the following formulation of the minimization problem:

$f *=\min _{f \in(V)}\left\{\frac{1}{p} \sum_{v \in V}\left\|\nabla f_{v}\right\|_{2}^{p}+\frac{\lambda}{2}\left\|f-f^{0}\right\|_{(V)}^{2}\right\}$,

where $p \in 0,+\infty$ is the smoothness degree and $\lambda$ is the fidelity parameter, called the Lagrange multiplier, which specifies the trade-off between the two competing terms. $\nabla f$ represents the weighted gradient of the function $f$ over the graph. The solution of Eq. (17) leads to a family of nonlinear filters parametrized by the weight function, the degree of smoothness, and the fidelity parameter.

The first energy in Eq. (17) is the smoothness term or regularizer, whereas the second is the fitting term. To solve the regularization problem, we use the Gauss-Jacobi iterative algorithm, where, for all $(u, v)$ in $E$ :

$$
\left\{\begin{array}{l}
f^{(0)}=f^{0} \\
\gamma^{(k)}(u, v)=w(u, v)\left(\left\|\nabla f^{(k)}(v)\right\|_{2}^{p-2}+\left\|\nabla f^{(k)}(u)\right\|_{2}^{p-2}\right) \\
f^{(k+1)}(v)=\frac{p \lambda f^{0}(v)+\sum_{u \sim v} \gamma^{(k)}(u, v) f^{(k)}(u)}{p \lambda+\sum_{u \sim v} \gamma^{(k)}(u, v)}
\end{array}\right.
$$

where $\gamma^{(k)}$ is the function $\gamma$ at the step $k$. The weights $w(u, v)$ are computed from $f^{0}$ or can be given as an input.

At each iteration, the new value $f^{(k+1)}$ at a vertex $v$ depends on two quantities: the original value $f^{0}(v)$ and a weighted average of the existing values in a neighborhood of $v$. We recall that the weighted gradient of the function $f$ in a vertex $v$ can be interpreted as the gradient magnitude at $v$. It may therefore be interpreted as the regularity of the function in the neighborhood of this vertex. It is defined as

$$
\|\nabla f(u)\|_{2}=\left[\sum_{v \sim u} w(u, v)\left(f_{v}-f_{u}\right)^{2}\right]^{1 / 2} .
$$

Figure 6(a) represents the projection of SIFT keypoints cloud over its three principal components in the diffusion space. The graph is built in this space and the new coordinates are classified. As we can see, the parameter $p$ considerably affects the result of the regularization. We observe the difference between Fig. 6(b) with $p=2$ and Fig. 6(c) with $p=0.5$. More simplification of the graph is obtained for $p<1$. In this case, the manifold shape is more clear and the classification process is improved when $p$ decreases.

\section{Multilabel Diffusion Algorithm}

\subsection{Graph-Based Segmentation Method}

Let $V$ be the set of all image points, $V_{L}=\left\{v_{k}\right\}_{k=1}^{m}$ be the set of labeled points (SIFT keypoints), and $V_{U}=$ $\left\{v_{u}\right\}_{u=m+1}^{N}$ be the set of unlabeled points. We extend the function $f(v)$ defined on the vertex $v$ (see Sec. 2.1) to

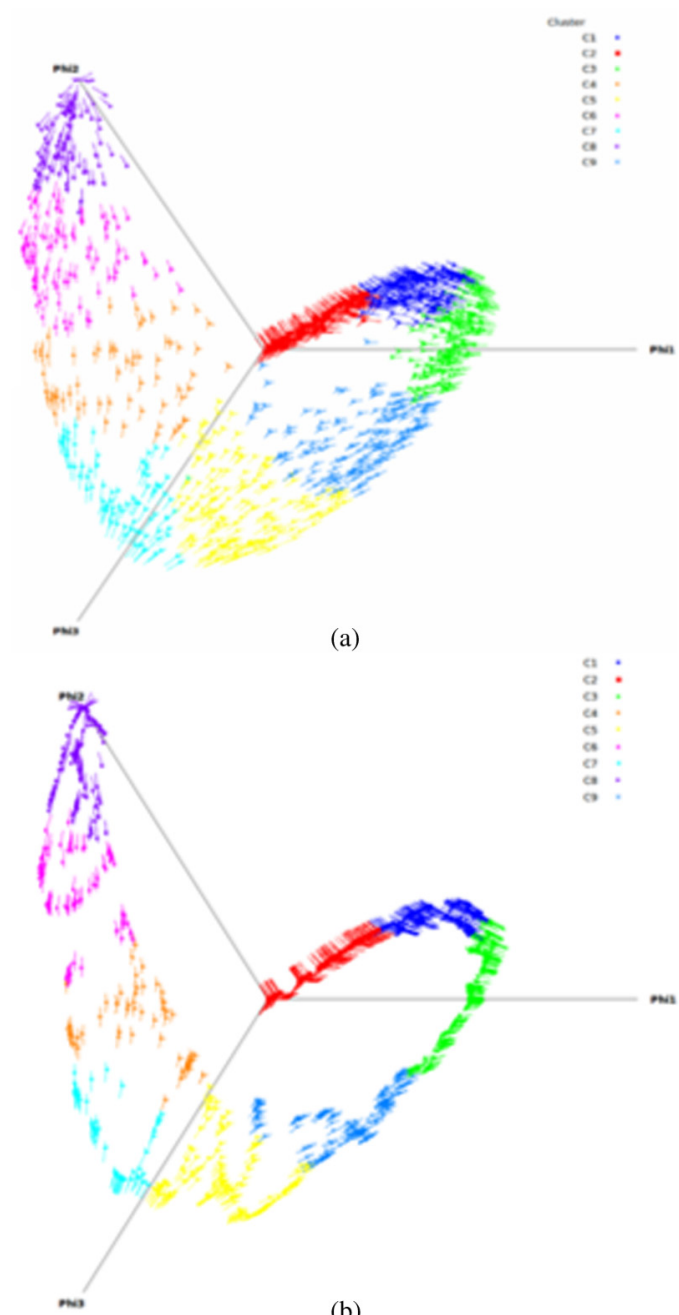

(b)

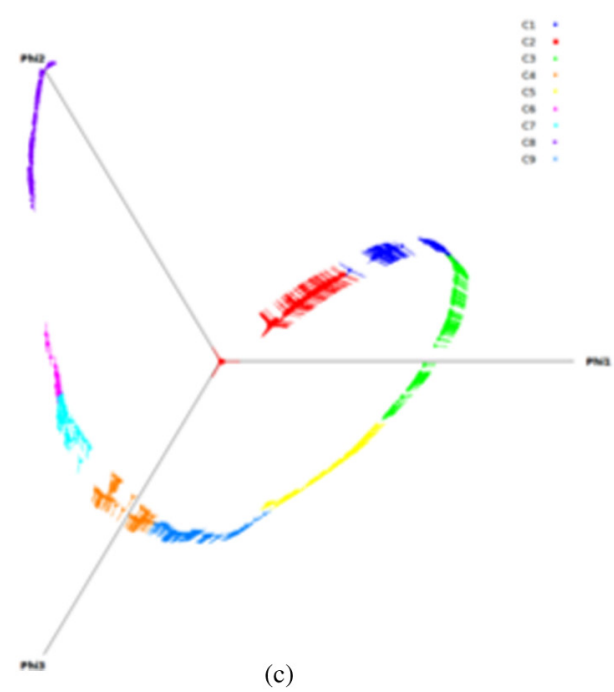

Fig. 6 Manifold denoising with difference $p$.

incorporate its label value $f_{0}=l \in \mathcal{L}=\{1,2, \ldots, c\}$. So $f$ will be represented by the tuple $\left\{f_{0}, f_{1}, \ldots, f_{K}\right\}$. To make the similarity between the graph vertices insensitive to $h_{i}$ (see Sec. 2.1), we normalize each $w(u, v)$ as follows: $w(u, v)=w(u, v) /\left[\max _{v \sim u} w(u, v)\right]$.

According to the theory of graph-based semisupervised learning, the label propagation can be formulated as the 
minimization of the energy function expressed in Eq. (17). Generally, we use $p=2$; then our strategy for propagating the labels can be formulated as an iterative process, where at every iteration step, only the labels of the unlabeled vertices are updated and the labels of the labeled ones will be clamped. For an unlabeled pixel $v$, its label at iteration $t$ will be computed by

$$
\left\{\begin{array}{l}
f=\left(f_{0}, f_{1}, \cdots, f_{K}\right) \\
f^{0}=f_{0} \in \mathcal{L} \\
f_{v}^{t+1}=\frac{1}{\lambda+\sum_{u \sim v} w(u, v)}\left[\lambda f_{v}^{0}+\sum_{u \sim v} w(u, v) f_{u}^{t}\right]
\end{array} .\right.
$$

If we set $\lambda=0$ and since $p(u, v)=w(u, v) /$ $\left[\sum_{v \sim u} w(u, v)\right]$ [see Eqs. (4) and (5)], one can define the propagation process from a vertex $u$ toward another vertex $v$ by

$f_{v}^{t+1}=\frac{1}{\sum_{u \sim v} w(u, v)} \sum_{u \sim v} w(u, v) f_{u}^{t}=\sum_{u \sim v} p(u, v) f_{u}^{t}$.

Then, Eq. (20) can be rewritten as

$$
\left\{\begin{array}{l}
f^{0}=f_{0} \in \mathcal{L} \\
f^{t+1}(v)=\sum_{u \sim v} p(u, v) f^{t}(u) \quad \forall v \in V^{.}
\end{array}\right.
$$

Then, the multilabel propagation procedure on the graph $G$ can be seen as a specific classification $\mathcal{C}$ on $V$ and considered as a function that assigns labels for each vertex $v$.

$f_{0}(v)=\underset{l \leq c}{\operatorname{argmax}} \mathcal{C}_{v l}$

Initially, let $\mathcal{C}_{v l}^{0}=1$ if $v$ is labeled as $l$ and $\mathcal{C}_{v l}^{0}=0$ otherwise. For unlabeled vertex $v, \mathcal{C}_{v l}^{0}=0$.

Therefore, Eq. (22) can be reformulated as

$$
f_{0}(v)=\underset{f_{0}(u) \leq c}{\operatorname{argmax}}\left[f_{0}(v)=f_{0}(u)\right] .
$$

The basic idea of our label propagation method is to consider an iterative algorithm where each node absorbs some label information from its neighborhood and updates its own label. This procedure will be repeated until all the nodes of the graph are labeled and not changed.

\subsection{Links with Other Methods}

Graph-based segmentation algorithms have been very successful in recent years. The modern variants are mainly built from a small set of basic algorithms: graph-cuts, random walk, and the shortest path algorithms. Recently, these three algorithms have been placed in a common framework that allows them to be considered as a special case of a general semisupervised segmentation algorithm with different choices of parameters $p$ and $q .{ }^{34}$

$$
\sum_{(u, v) \in E}\left[w(u, v)^{p}\left|f_{u}-f_{v}\right|\right]^{q}
$$

where $w(u, v)$ is a function that measures the interactions between the nodes of the graph and $\left|f_{u}-f_{v}\right|$ measures the distance between them. Thus, our Eq. (21) can be easily driven from this framework if we pose $p=q=1$ and $p(u, v) f^{t}(v)=w(u, v)\left|f_{u}-f_{v}\right|$. Furthermore, a connection between Eq. (21) and the energy minimization by Markov random field (MRF) models can also be established. Recall that an MRF is often described by a set of $V$ vertices along with a neighborhood on them. On each vertex $v$, there is a random variable $f(v)$, which can take values from a finite set (e.g., $f(v) \in \mathcal{L}=\{1,2, \ldots, c\}$ ). The goal is to find $f *$ that satisfies

$f *=\operatorname{argmin}\left\{\sum_{v} \phi[f(v)]+\sum_{u \sim v} \phi_{u v}[f(u), f(v)]\right\}$.

$\phi[f(v)]$ is a function on the variable $f(v)$ and can be defined as a likelihood energy where

$\phi[f(v)]= \begin{cases}\infty & \text { if } f^{t+1}(v) \neq f^{t}(v) \\ & (\text { i.e., the label of } v \text { will be changed }) \\ 0 & \text { otherwise }\end{cases}$

and $\phi_{u v}[f(u), f(v)]$ measures the information exchange between the labeled vertex $u$ and the unlabeled vertex $v$. It can, in turn, be defined by referring to Eqs. (21) and (24) as $\phi_{u v}[f(u), f(v)]=p(u, v)|f(u)-f(v)| . p(u, v)$ is the probability corresponding to the random walk from $u$ toward $v$. Since $v$ has to be labeled, $f(v)=\infty$; then the minimization of Eq. (25) is equivalent to solving the following optimization problem:

$\min _{f(u) \in \mathcal{L}}\left\{\sum_{u \sim v} p(u, v)|f(u)-f(v)|\right\}$.

Thus, we have shown that our method can be derived from the framework of energy minimization of MRF.

\section{Experiments}

We conducted our experiments on a collection of images issued from the Berkeley database. ${ }^{35}$ First, SIFT keypoints are located on each image; then a visual similarity graph is constructed over these keypoints. Each vertex represents an SIFT keypoint and a weighed edge measures the similarity between two connected vertices $u$ and $v$ by using the Gaussian kernel $w_{u v}$ [Eq. (3)]. $F_{(.)}$is the histogram of the patch surrounding the considered point expressed in the LAB color space. Many variations of distances $\left\|F_{u}-F_{v}\right\|^{2}$ can be used, including the Bhattacharya, Kolmogorov, intersection, and correlation distances. ${ }^{36}$

Once the similarity between keypoints is defined, the eigendecomposition of the underlying Laplacian matrix leads to the definition of a new reduced pose space, where each SIFT keypoint is expressed by new Euclidean coordinates calculated according to its surrounding patch.

Figure 7 shows some classification results on the keypoints cloud projected in the Euclidean pose space and their projections on the input images. The graphs in Fig. 7(b) represent the regularized versions of those in Fig. 7(a) with $p=2$. As we can see, this has highly made in evidence the object of interest class and the foreground class [see also Fig. 7(c)]. Figure 7(d) shows object/foreground 
(a)

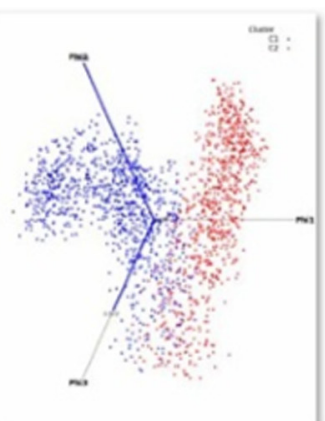

(b)

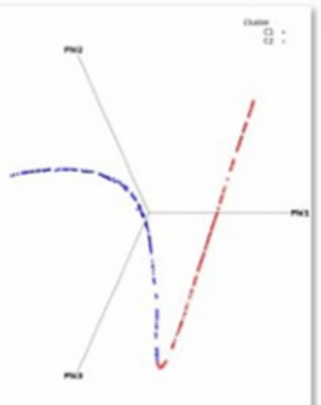

(c)
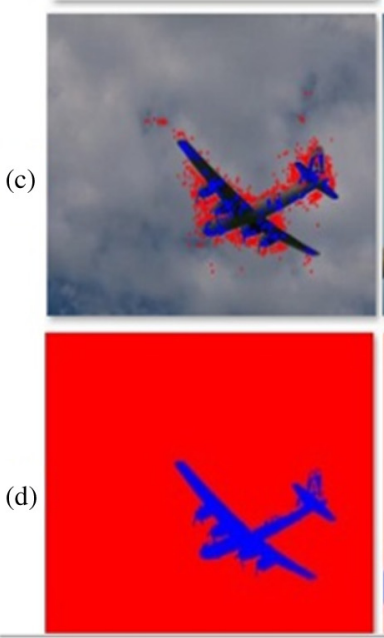
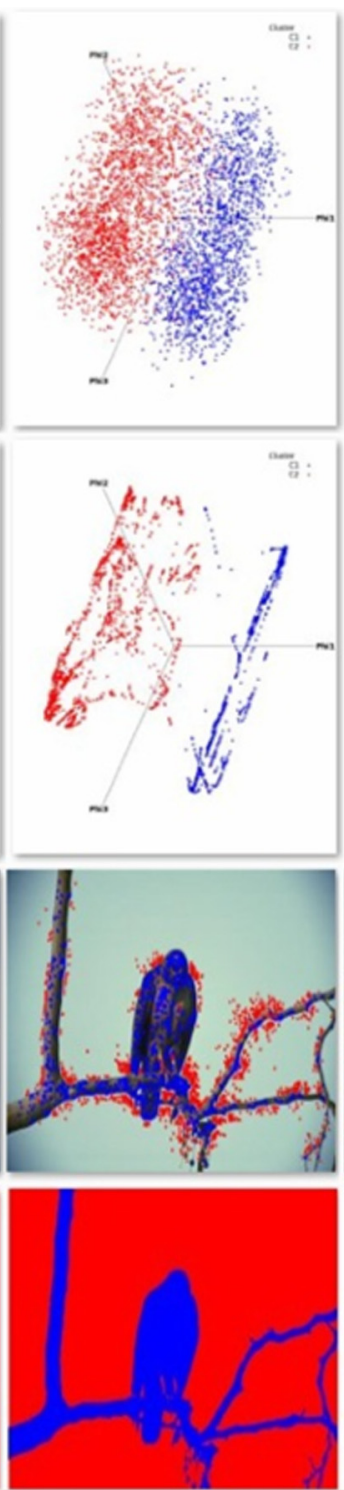
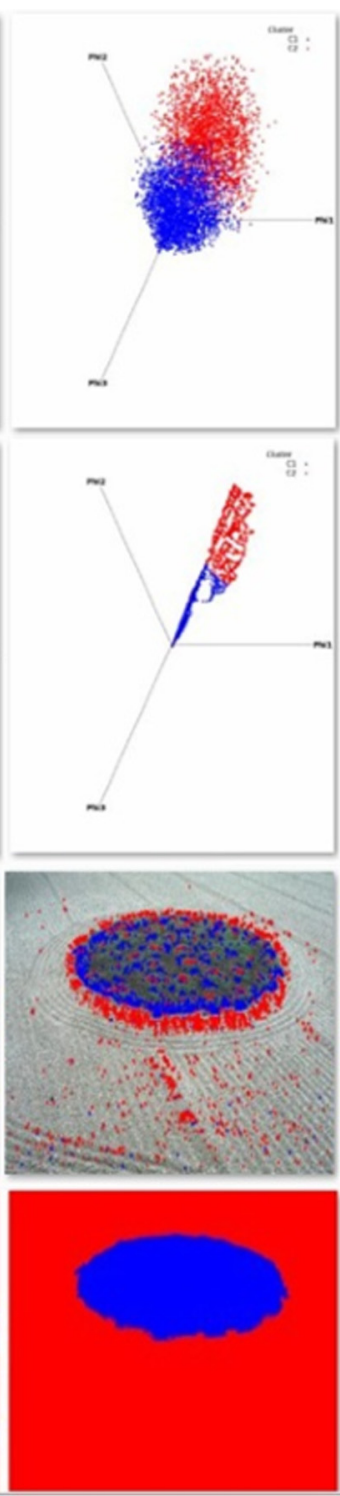
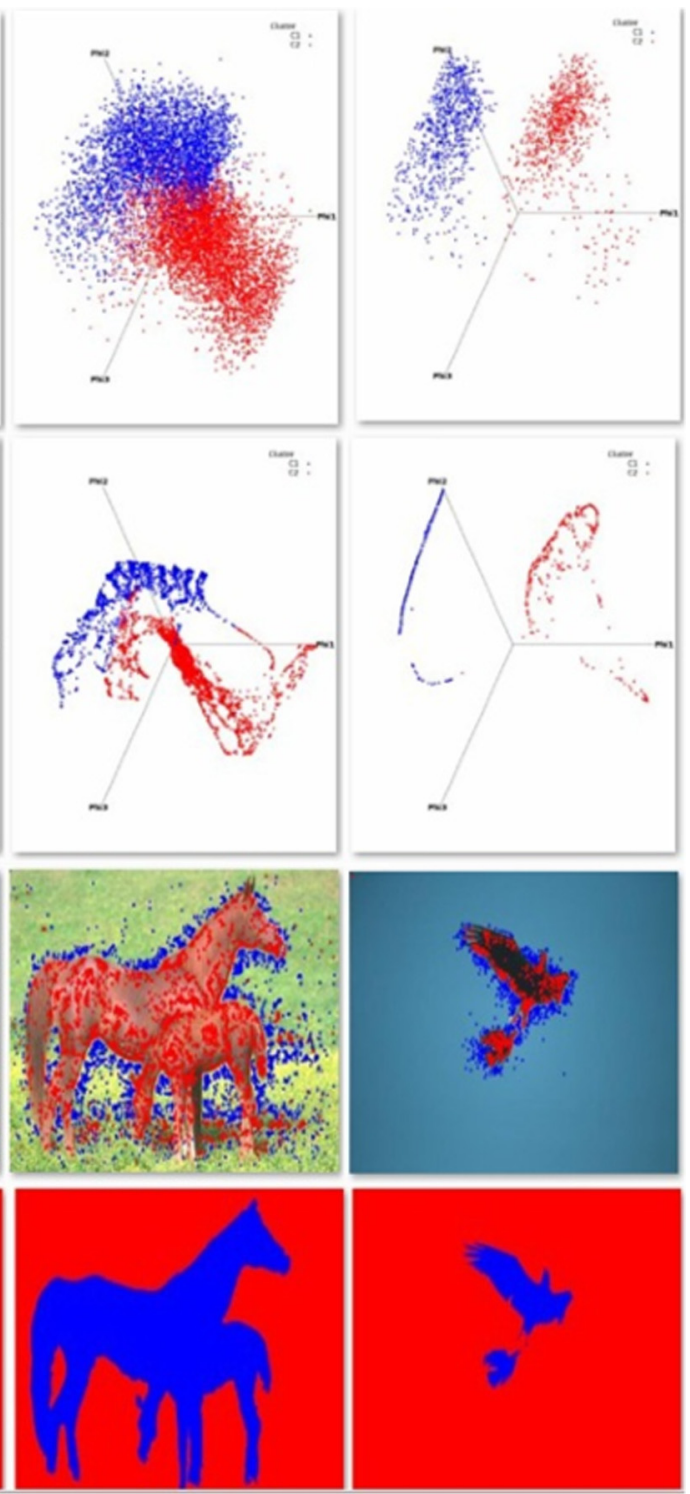

Fig. 7 Keypoints classification and their projections on the reduced space and the input images.

segmentation corresponding to the seeded images in Fig. 7(c). These labels are propagated on the corresponding image graphs to extract the object of interest and obtained after 100 iterations using Eq. (21).

The F-measure, recall, and precision computed as described in Ref. 37 and corresponding to these images are shown in Table 1 (image1, image2, image3, image4, and image5 correspond to the images in Fig. 7(c) from left to right, respectively).

Figure 8 presents another example of multilabel image segmentation. In Fig. 8(a), SIFT keypoint classes are identified by using spectral embedding into a Euclidean manifold. These classes are better separated through manifold denoising with $p=2$ [Fig. 8(b)]. The projections of these classes on the images are presented in Figs. 8(c) and $8(\mathrm{~d})$.

To assess the performance of our framework, we used two objective segmentation measures: the Rand index (RI) and the global consistency error (GCE). The RI measures the consistency of a labeling between a given segmentation and its corresponding ground truth by using the ratio of pairs of pixels having the same labels. The goal is to assign two pixels to the same class if and only if they are similar in order to measure the percentage of similarity. The GCE measures the extent for which one segmentation can be viewed as a refinement of the other one. It is worth mentioning that the similarity measure RI is better when it is higher and the distance measure GCE is better when it is lower. Often, GCE favors oversegmentation. Hence, to compare with other methods,

Table 1 F-measure, recall, and precision.

\begin{tabular}{llllll}
\hline & Image 1 & Image2 & Image3 & Image4 & Image5 \\
\hline F-measure & 0.8669 & 0.9211 & 0.9743 & 0.5937 & 0.9024 \\
Recall & 0.9911 & 0.9718 & 0.9734 & 0.4532 & 0.9996 \\
Precision & 0.7703 & 0.8754 & 0.9751 & 0.8606 & 0.8225 \\
\hline
\end{tabular}


(a)

(b)

(c)

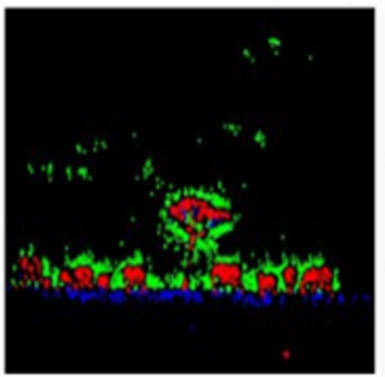

(d)

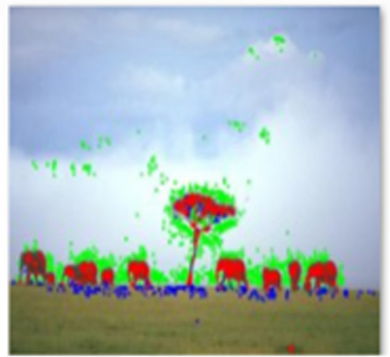

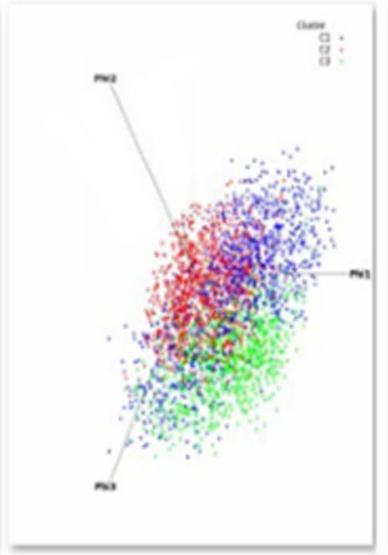
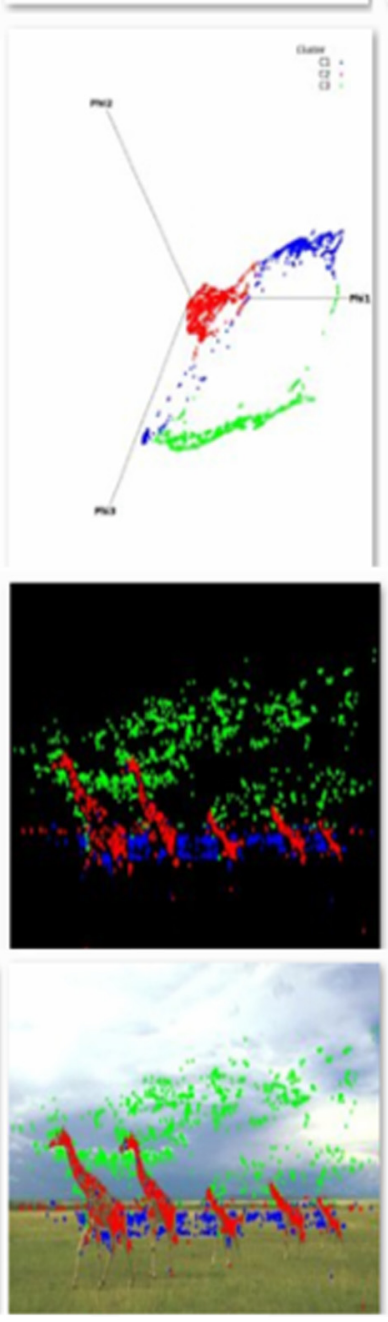
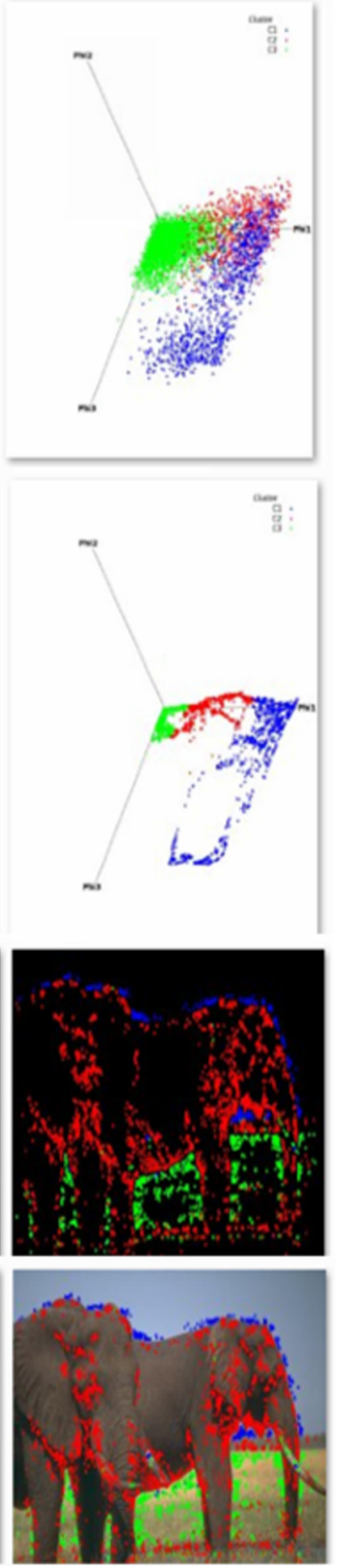

Fig. 8 Multilabel image segmentation.

we have performed the segmentation without considering regions of an area $<2 \%$ of the image.

A comparative evaluation of our method with four wellknown ones, namely fuzzy C-means algorithm, ${ }^{38}$ WaterShed algorithm, ${ }^{39}$ normalized cuts, ${ }^{40}$ and the mean-shift algorithm, ${ }^{41}$ is implemented by using the library Pandore. ${ }^{42}$

We recall that the mean shift implementation performs clustering in a five-dimensional space with two spatial and three color dimensions. Note that the kernel width has a very important effect on the algorithm performance. However, the choice of an appropriate value for the kernel width is still an open problem. In the present experiments, the spatial parameter $h s$ is set to 10 and the range (color) kernel bandwidth was fixed to 20 .

Figure 9 shows qualitative results of our algorithm applied on the same images in Fig. 7. It can be observed that when the seeds are well dispersed on the image, the segmentations have a closer similarity with the human one. 


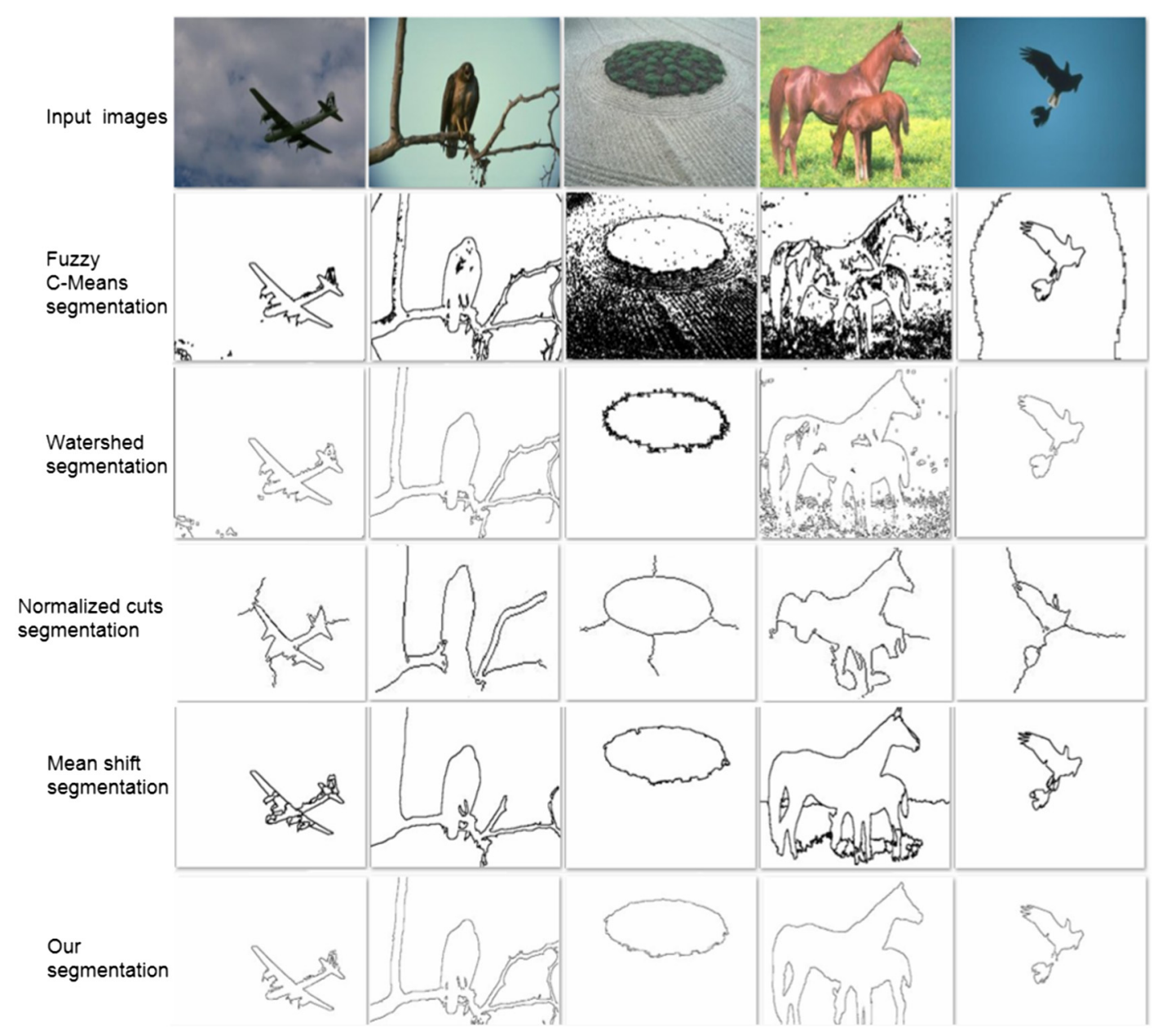

Fig. 9 The performance comparison with several segmentation approaches.

Quantitative results of our experiments are summarized by the histograms in Figs. 10 and 11 . These results clearly show that the original images and the results obtained by our approach are very close. Indeed, most of the GCE values found are $<0.19$, while a larger number of RI values are grouped below 0.8. The peaks found for GCE and for $\mathrm{RI}$ are 0.18 and 0.89 , respectively. The corresponding segmented images are very similar visually and quantitatively.

It can be seen from the $x$ axis that the poor performance of segmentation from the GCE and the RI point of view are 0.01 and 0.53 , respectively. This includes images that are difficult

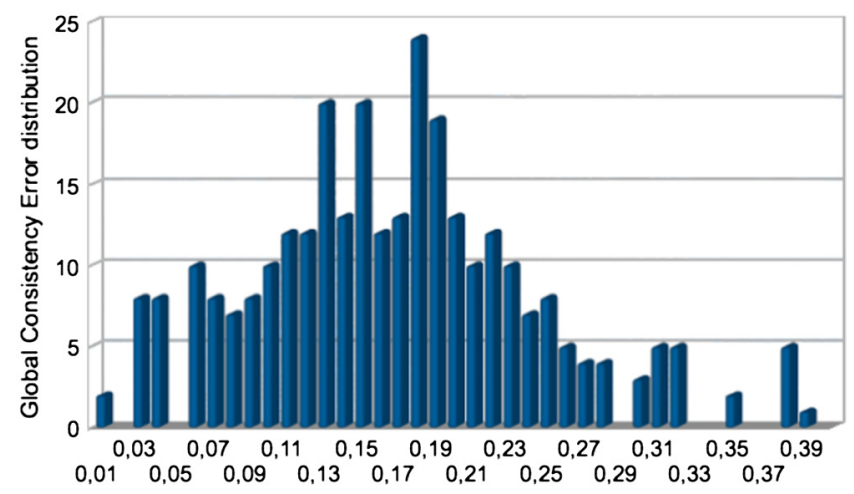

Fig. 10 Distribution of the global consistency error. to segment. This can be explained by the problem of borders and by the choice of segmentation parameters.

Table 2 presents the performance evaluation of our method compared with the state-of-art ones. As it can be observed, our method produces better results. It gives the lowest measure of GCE and the highest mean RI score.

The proposed method gives better results by producing a fewer number of homogeneous regions. Also, it provides a good solution to overcome the sensitiveness to the initialization condition of clusters. The oversegmentation is decreased effectively since this method integrates diffusion with automatic seeded region growing.

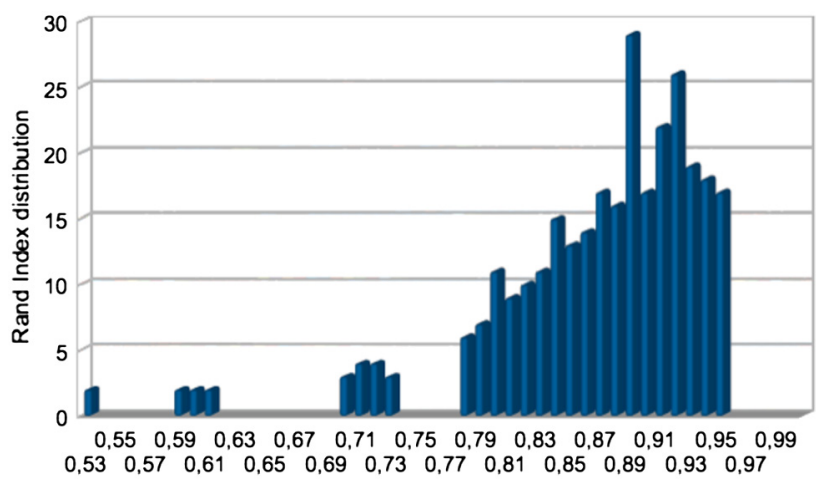

Fig. 11 Distribution of the Rand index. 
Table 2 Performance evaluation of our algorithm.

\begin{tabular}{lcc} 
& Global consistency error & Rand index \\
\hline Ground truth (human) & 0.079 & 0.875 \\
Fuzzy C-means & 0.221 & 0.789 \\
Watershed & 0.203 & 0.697 \\
Normalized cuts & 0.218 & 0,723 \\
Mean-shift & 0.259 & 0.755 \\
Our approach & $\mathbf{0 . 1 8 9}$ & $\mathbf{0 . 7 9 9}$ \\
\hline
\end{tabular}

Note: Bold values show the results obtained by our approach.

\section{Conclusions}

In this paper, we have addressed the problem of learning from a small informative set using a graph-based diffusion model. A case study was automatic image segmentation. We presented a unified framework through three steps: a spectral graph embedding of SIFT keypoints, manifold denoising with $p$-Laplacian, and a multilabel diffusion algorithm. With this scheme, a set of keypoints are automatically located on the image and, subsequently, distributed over the background and the regions of interest (ROIs). Thereafter, these seeds are propagated progressively on the graph, representing the image, which exploits the acquired semantic information and visual features among pixels until the segmentation of the ROI. We implemented the proposed framework and obtained encouraging experimental results. The proposed method produces good boundaries with respect to the ground-truth segmentation and relatively higher precision compared with other methods. Nevertheless, the number of classes needs to be specified in advance.

We currently explore the possibility of generalizing the concept of this framework for video segmentation by considering three-dimensional images representing the video keyframes and incorporating audio features to help our categorization.

\section{Acknowledgments}

This paper is supported by the PRETHERM ANR project No. ANR-09-BLAN-0352, co-financed by the French National Research Agency (ANR). The authors wish to thank the reviewers, the associate editor, and the editor-inchief for their helpful suggestions and comments to improve this paper. We also would like the thank Professor Abder Elmoataz for discussions about the discrete $p$-regularization and its constructive remarks.

\section{References}

1. Y. Boykov and M. Jolly, "Interactive graph cuts for optimal boundary \& region segmentation of objects in n-d images," in Proc. 8th Intl. Conf. on Computer Vision, pp. 105-112, IEEE Computer Society (2001).

2. V. Kolmogorov and R. Zabih, "What energy functions can be minimized via graph cuts," IEEE Trans. Pattern Anal. Mach. Intell. 26(2), 65-81 (2004).

3. G. Brunner et al., "Patch-cuts: a graph-based image segmentation method using patch features and spatial relations," in Proc. of the British Machine Vision Conf., F. Labrosse, pp. 29.1-29.11, BMVA Press (2010).
4. B. L. Price, B. Morse, and S. Cohen, "Geodesic graph cut for interactive image segmentation," in IEEE Conf. on Computer Vision and Pattern Recognition, pp. 3161-3168, IEEE Computer Society (2010).

5. N. D. F. Campbell et al., "Automatic 3D object segmentation in multiple views using volumetric graph-cuts," IVC J. 28(1), 14-25 (2010).

6. J.-S. Kim and K.-S. Hong, "A new graph cut-based multiple active contour algorithm without initial contours and seed points," MVA J. 19(3), 181-193 (2008).

7. C. Rother, V. Kolmogorov, and A. Blake, "Grabcut: interactive foreground extraction using iterated graph cuts," ACM Trans. Graph. 23(3), 309-314 (2004).

8. N. Xu, N. Ahuja, and R. Bansal, "Object segmentation using graph cuts based active contours," Comput. Vis. Image Underst. 107(3), 210-224 (2007).

9. A. Blake et al., "Interactive image segmentation using an adaptive GMMRF model," in Proc. European Conf. in Computer Vision, pp. 428-441, Springer (2004).

10. J. Wang, M. Agrawala, and M. F. Cohen, "Soft scissors: an interactive tool for realtime high quality matting," ACM Trans. Graph. 26(3), 9 (2007).

11. C. Harris and M. Stephens, "A combined corner and edge detector," in Proc. of the Alvey Vision Conf., C. J. Taylor, Ed., pp. 147-151, Alvety Vision Club (1988).

12. D. G. Lowe, "Distinctive image features from scale invariant keypoints," Int. J. Comput. Vis., 60(2), 91-110 (2004).

13. K. Mikolajczyk and C. Schmid, "A performance evaluation of local descriptors," IEEE Trans. Pattern Anal. Mach. Intell. 27(10), 16151630 (2005).

14. H. Bay, T. Tuytelaars, and L. Van Gool, "SURF: speeded up robust features," Int. J. Comput. Vis. Image Underst. 110(3), 346-359 (2008).

15. J.-M. Morel and G. Yu, "ASIFT: a new framework for fully affine invariant image comparison," SIAM J. Imaging Sci. 2(2), 438-469 (2009).

16. Y. Ke and R. Sukthankar, "PCA-sift: a more distinctive representation for local image descriptors," in Proc. of the IEEE Computer Society Conf. on Computer Vision and Pattern Recognition, pp. 506-513, IEEE Computer Society (2004).

17. S. Lafon and A. B. Lee, "Diffusion maps, and coarse-graining: a unified framework for dimensionality reduction, graph partitioning, and data set parameterization," IEEE Trans. Pattern Anal. Mach. Intell. 28(9), 1393-1403 (2006).

18. G. W. Stewart and J.-g. Sun, Matrix Perturbation Theory, Academic Press, Boston (1990).

19. L. Ren-Cang, "On perturbations of matrix pencils with real spectra, a revisit," Math. Comput. 72(242), 715-728 (2003).

20. E. J. Nyström, "Über die praktische Auflösung von Integralgleichungen mit Anwendungen auf Randwertaufgaben," Acta Mathematica 54(1), 185-204 (1930).

21. C. T. H. Baker, The Numerical Treatment of Integral Equations, Clarendon Press, Oxford (1977).

22. A. Robles-Kelly, S. Sarkar, and E. R. Hancock, "A fast leading eigenvector approximation for segmentation and grouping," in Proc. 16th Int. Conf. on Pattern Recognition, pp. 639-642, IEEE Computer Society (2002).

23. L. Huang et al., "Spectral clustering with perturbed data," in Advances in Neural Information Processing Systems, pp. 705-712, Curran Associates, Inc. (2008).

24. C. Williams and M. Seeger, "Using the Nyström method to speed up kernel machines, in Advances in Neural Information Processing Systems, pp. 682-688, MIT Press (2001).

25. C. Fowlkes et al., "Spectral grouping using the Nyström method," IEEE Trans. Pattern Anal. Mach. Intell. 26(2), 214-225 (2004).

26. J. C. Platt, "FastMap, MetricMap, and Landmark MDS are all Nyström algorithms," in 10th Int. Workshop on Artificial Intelligence and Statistics, pp. 261-268 (2005).

27. K. Zhang and J. T. Kwok, "Density-weighted Nystrom method for computing large kernel eigensystems," Neural Comput. 21(1), 121-146 (2009).

28. R. B. Cattell, "The scree test for the number of factors," Multivariate Behav. Res. 1(2), 245-276 (1966).

29. G. Raiche, M. Riopel, and J. G. Blais, "Non graphical solutions for the Cattell's scree test," in Proc. Int. Annual Meeting of the Psychometric Society, Montreal, Canada (2006).

30. S. Lafon, Y. Keller, and R. R. Coifman, "Data fusion and multicue data matching by diffusion maps," IEEE Trans. Pattern Anal. Mach. Intell. 28(11), 1784-1797 (2006).

31. B. Nadler et al., "Diffusion maps - a probabilistic interpretation for spectral embedding and clustering algorithms," in Principal Manifolds for Data Visualization and Dimension Reduction, A. N. Gorban et al., Eds., Vol. 58, pp. 238-260, Springer, Berlin, Heidelberg (2007).

32. M. Ghoniem, Y. Chahir, and A. Elmoataz, "Nonlocal video denoising, simplification and inpainting using discrete regularization on graphs," J. Signal Process. 90(8), 2445-2455 (2010).

33. A. Elmoataz, O. Lezoray, and S. Bougleux, "Nonlocal discrete regularization on weighted graphs: a framework for image and manifold processing," IEEE Trans. Image Process. 17(7), 1047-1060 (2008). 
34. A. Sinop and L. Grady, "A seeded image segmentation framework unifying graph cuts and random walker which yields a new algorithm," in IEEE 11th Int. Conf. on Computer Vision, pp. 1-8, IEEE Computer Society (2007).

35. D. Martin et al., "A database of human segmented natural images and its application to evaluating segmentation algorithms and measuring ecological statistics," in Proc. 8th Int'l Conf. on Computer Vision, Vol. 2, pp. 416-423, IEEE Computer Society (2001)

36. L. Ballan et al., "Video event classification using string kernels," Multimed. Tools Appl. 48(1), 69-87 (2010).

37. M. Kulkarni and F. Nicolls, "Interactive image segmentation using graph cuts," in Twentieth Annual Symp. of the Pattern Recognition Association of South Africa, pp. 99-104 (2009).

38. J. C. Bezdek, R. Ehrlich, and W. Full, "FCM: the fuzzy c-means clustering algorithm," J. Comput. Geosci. 10(2-3), 191-203 (1984).

39. L. Vincent and P. Soille, "Watersheds in digital spaces: an efficient algorithm based on immersion simulations," IEEE Trans. Pattern Anal. Mach. Intell. 13(6), 583-598 (1991).

40. J. Shi and J. Malik, "Normalized cuts and image segmentation," IEEE Trans. Pattern Anal. Mach. Intell. 22(8), 888-905 (2000).

41. D. Comaniciu and P. Meer, "Mean shift: a robust approach toward feature space analysis," IEEE Trans. Pattern Anal. Mach. Intell. 24(5), 603-619 (2002)

42. "Pandore: Une bibliothèque d'opérateurs de traitement d'images," Version 6.6, https://clouard.users.greyc.fr/Pandore (2013).
Youssef Chahir is a professor in the computer science department at Lower Normandy University. He is a member of the Image Team at the GREYC Laboratory. His research interest fields include image and video processing and analysis, multimedia data mining, spectral analysis and restitution, and animation in virtual environments.

Abderraouf Bouziane is an associate professor at the computer science department at Bordj Bou Arreridj University. He is a member of the MSE Laboratory. He worked as an invited researcher at GREYC Laboratory. His research interest fields include spectral analysis, organization, and indexing of high-dimensional multimedia data.

Messaoud Mostefai is an associate professor at the computer science department at Bordj Bou Arreridj University. He is a member of the MSE Laboratory. His main research interests are focused on classification and biometric identification, computer vision and signal processing.

Adnan Al Alwani is a PhD student at the Image Team, Lower Normandy University. His research interest fields include pattern recognition, signal, image, and video processing. 